\title{
Extended Coherence Time on the Clock Transition of Optically Trapped Rubidium
}

\author{
G. Kleine Büning, ${ }^{1, *}$ J. Will, ${ }^{1}$ W. Ertmer, ${ }^{1}$ E. Rasel,,${ }^{1}$ J. Arlt, ${ }^{2}$ and C. Klempt ${ }^{1}$ \\ ${ }^{1}$ Institut für Quantenoptik, Leibniz Universität Hannover, Welfengarten 1, 30167 Hannover, Germany \\ ${ }^{2}$ QUANTOP, Institut for Fysik og Astronomi, Aarhus Universitet, Ny Munkegade 120, 8000 Aarhus C, Denmark \\ F. Ramirez-Martinez, ${ }^{3, \dagger}$ F. Piéchon, ${ }^{4}$ and P. Rosenbusch ${ }^{3}$ \\ ${ }^{3}$ LNE-SYRTE, Observatoire de Paris, CNRS, UPMC, 61 av de l'Observatoire, 75014 Paris, France \\ ${ }^{4}$ Laboratoire de Physique des Solides, CNRS, UMR 8502, Univ. Paris-Sud, 91405 Orsay, France
}

(Received 11 March 2011; published 16 June 2011)

\begin{abstract}
Optically trapped ensembles are of crucial importance for frequency measurements and quantum memories but generally suffer from strong dephasing due to inhomogeneous density and light shifts. We demonstrate a drastic increase of the coherence time to $21 \mathrm{~s}$ on the magnetic field insensitive clock transition of ${ }^{87} \mathrm{Rb}$ by applying the recently discovered spin self-rephasing [C. Deutsch et al., Phys. Rev. Lett. 105, 020401 (2010)]. This result confirms the general nature of this new mechanism and thus shows its applicability in atom clocks and quantum memories. A systematic investigation of all relevant frequency shifts and noise contributions yields a stability of $2.4 \times 10^{-11} \tau^{-1 / 2}$, where $\tau$ is the integration time in seconds. Based on a set of technical improvements, the presented frequency standard is predicted to rival the stability of microwave fountain clocks in a potentially much more compact setup.
\end{abstract}

DOI: 10.1103/PhysRevLett.106.240801

PACS numbers: 06.30.Ft, 03.65.Yz, 03.75.Dg, 67.85.-d

Atomic clocks provide unprecedented precision in the determination of time. Most prominently, the SI second is experimentally determined by measuring the ${ }^{133} \mathrm{Cs}$ hyperfine transition with microwave atomic fountain clocks [1]. The ongoing quest for the best definition of time is currently led by optical frequency standards [2,3]. Moreover, applications ranging from the determination of fundamental constants to navigation and communication technology demand more compact frequency standards. In contrast to microwave fountain clocks, optically trapped atomic ensembles [4] promise high stability in compact setups due to long interrogation times. However, they typically suffer from a short coherence time.

In addition to frequency measurements, trapped ensembles serve as efficient quantum memories for long-distance quantum communication [5]. In a series of experiments, deterministic photons were produced [6], entanglement between photons and spin waves was created $[7,8]$, and even prototype networks for entanglement distribution were demonstrated $[9,10]$. By employing traps instead of ballistic ensembles, the memory lifetime was already extended from microseconds [6] to milliseconds [11,12]. Longer coherence times would also be beneficial in this field.

The coherence time is limited by dephasing due to trapinduced frequency shifts which are necessarily inhomogeneous. There have been several attempts to overcome the influence of these shifts [13]. Optical lattice clocks employ a dipole trap at a magic wavelength to suppress the differential light shift (DLS) [14]. Magic wavelengths for microwave transitions have been predicted for $\mathrm{Al}$ and Ga [15]. However, the microwave transitions in alkali atoms such as $\mathrm{Rb}$ and $\mathrm{Cs}$ do not exhibit magic wavelengths [16] unless traps of particular polarization combined with a specific magnetic field are employed [17,18]. There have been approaches to increase the coherence time by collisional spectral narrowing [19] or artificial rephasing through a sequence of microwave pulses [20]. A further approach is the use of a magnetic confinement for which the inhomogeneity of the Zeeman shift is canceled by the density shift [21]. In such a trap, it was shown that the exchange interaction can be used to induce spin selfrephasing (SSR) [22]. It is therefore an important question whether this effect may also be used for the magnetically insensitive clock states.

In this Letter, we employ spin self-rephasing to extend the coherence time of optically trapped atoms to $21 \mathrm{~s}$. We show that both the inhomogeneity of the DLS and the inhomogeneity of the density shift can be overcome by SSR. Within our investigation, we perform Ramsey spectroscopy using the magnetically insensitive clock states of the ${ }^{87} \mathrm{Rb}$ hyperfine transition, a secondary representation of the second. Thus, we evaluate the applicability of SSR for atomic microwave frequency standards and demonstrate a stability of $2.4 \times 10^{-11} \tau^{-1 / 2}$. For a purpose-built apparatus with standard technical improvements, we predict a 300fold stability enhancement, primarily limited by temperature fluctuations. Thereby our technique enters the stability range of most atomic fountain clocks [1] in a potentially more compact setup. Our findings prove the fundamental nature of SSR which is independent of the type of trap and the magnetic quantum number. Thus, its applicability extends to optical lattice clocks as well as quantum information with atomic ensembles. 
The SSR inhibits dephasing and induces characteristic contrast revivals, mediated by the exchange interaction. This effect can be understood as follows. Each atom in a superposition of the two clock states can be represented by an effective spin $1 / 2$. The exchange interaction of two atoms can be described within the singlet-triplet basis $[23,24]$. The atom pair, initially in the triplet state, dephases by Rabi flopping towards the singlet state. For bosons (fermions), the interaction energy shifts solely the triplet (singlet) state due to the symmetry of the spatial wave function. The shift tunes the states out of resonance and thereby suppresses the Rabi flopping and hence the dephasing. The effect can also be visualized in the Bloch sphere representation, where the exchange interaction corresponds to a rotation of two individual spins around their sum [25]. In the following, we apply the quantitative model developed in Ref. [22]. In this approach, the atoms are divided into classes of constant energy and a mean spin for each class is considered.

Two conditions have to be met for SSR to occur: (i) the inhomogeneity of the transition frequency, i.e., the dephasing rate, must be smaller than the exchange rate, $\Delta_{0}<\omega_{\text {ex }}$, and (ii) the rate of velocity changing collisions must be smaller than the exchange rate so that rephasing establishes before atoms change energy class, $\gamma_{c}<\omega_{\mathrm{ex}} / \pi$. These three parameters can be predicted for a thermal gas in an harmonic potential. We use the definition of $\Delta_{0}, \gamma_{c}$, and $\omega_{\text {ex }}$ from [22]. In our case, the dephasing rate $\Delta_{0}$ is composed of two inhomogeneous frequency shifts, the differential light shift and the density shift in the meanfield approximation: $\Delta_{0}=k_{B} T / 2 \hbar \times \delta \alpha / \alpha-\gamma \bar{n} / 4$. Here, $\delta \alpha$ and $\alpha$ denote the differential and the total light shift per intensity, $T$ is the ensemble temperature, $\bar{n}$ the mean density, and $\gamma=\frac{4 \pi \hbar}{m}\left(a_{\uparrow}-a_{\downarrow \downarrow}\right)$, where $m$ is the mass and $a_{\uparrow}, a_{\downarrow}$ are the scattering lengths between atoms in the same state.

In a first set of experiments, we explore the onset of SSR in an optically trapped ensemble. Our experimental apparatus has been described in detail in Ref. [26]. An optical dipole trap at $\lambda=1064 \mathrm{~nm}$ is provided by two intersecting laser beams with beam waists of 60 and $75 \mu \mathrm{m}$, yielding a mean trap frequency of $2 \pi \times 70 \mathrm{~Hz}$. A cloud of ${ }^{87} \mathrm{Rb}$ atoms is prepared in the state $|\downarrow\rangle=\left|F=1, m_{F}=0\right\rangle$ at a temperature of 400(20) nK with a variable mean density $\bar{n}$ from 0.18 to $1.38 \times 10^{12} \mathrm{~cm}^{-3}$. The atoms are held in the trap for a constant time, during which Ramsey spectroscopy of the clock transition to $|\uparrow\rangle=\left|F=2, m_{F}=0\right\rangle$ is performed. The Ramsey sequence with a variable interrogation time of $0-1 \mathrm{~s}$ is placed at the end within this hold time. The coupling is realized by two microwave $\pi / 2$ pulses of $100 \mu$ s duration, generated by a low phase noise synthesizer [27] locked to a passive hydrogen maser. After the Ramsey sequence, the trap is switched off to allow for ballistic expansion and Stern-Gerlach separation of the two states. The number of atoms in both states, $N_{\uparrow}$ and $N_{\downarrow}$, is detected by simultaneous absorption imaging, calibrated according to Ref. [28].

Figure 1 shows the population transfer $P=N_{\uparrow} /\left(N_{\uparrow}+N_{\downarrow}\right)$ as a function of the interrogation time for four different densities. Each subplot shows clear sinusoidal Ramsey fringes with a period of $\approx 45 \mathrm{~ms}$, in agreement with a microwave detuning of $15 \mathrm{~Hz}$ and residual shifts of $-7 \mathrm{~Hz}$ (see below). For the lowest density, the contrast of the Ramsey fringes drops within the first $500 \mathrm{~ms}$ due to dephasing. However, the contrast does not decay completely within $1 \mathrm{~s}$, as expected for pure dephasing, indicating the presence of SSR. At higher mean density, a revival of the contrast emerges and a considerably longer coherence time is observed. As the density is increased further, the contrast revivals appear earlier, since the higher exchange rate $\omega_{\text {ex }}$ ensures faster rephasing. The contrast does not recover fully at later times, because the rate of velocity changing collisions $\gamma_{c}$ abates rephasing by a mixing of trap states.

The measured contrast is well reproduced by the numerical model of Ref. [22], where $\Delta_{0}, \omega_{\mathrm{ex}}$, and $\gamma_{c}$ are used as free fitting parameters. They agree within $25 \%$ with their prediction if the same correction factors of 1.6 for $\Delta_{0}$ and

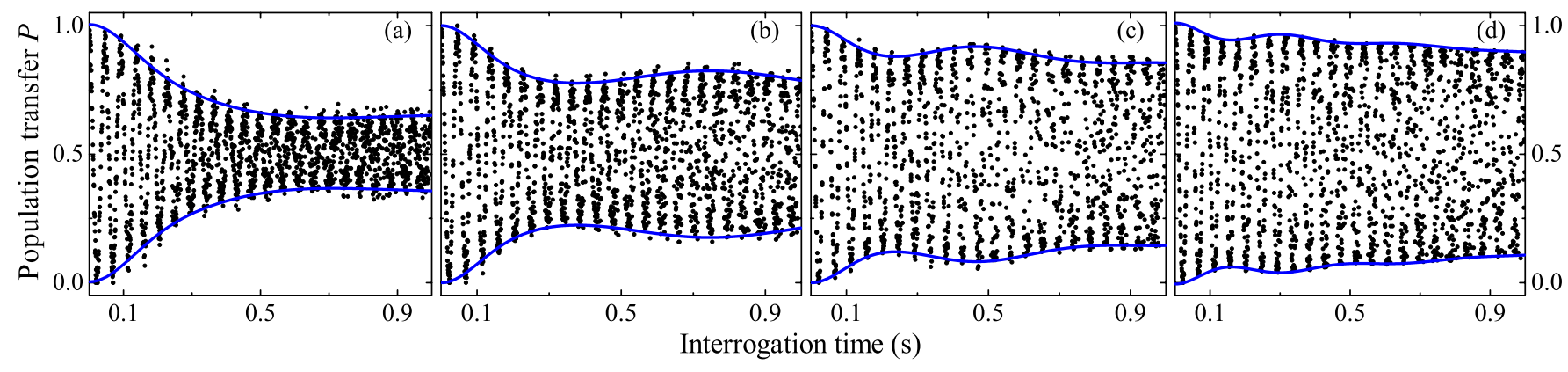

FIG. 1 (color online). Population transfer $P=N_{\uparrow} /\left(N_{\uparrow}+N_{\downarrow}\right)$ after a Ramsey sequence as a function of the interrogation time. The mean density increasing from (a) to (d) is $\{0.18,0.36,0.65,1.38\} \times 10^{12} \mathrm{~cm}^{-3}$. Both the increase of the coherence time and the characteristic contrast revivals due to spin self-rephasing are clearly visible. Further revivals at longer interrogation times are damped out due to a mixing of trap states. Both conditions for spin self-rephasing are fulfilled: $\omega_{\text {ex }} / \Delta_{0}$ increases with density from 4.3 to 23 , giving rise to the observed onset of rephasing, while $\omega_{\text {ex }} /\left(\pi \gamma_{c}\right)=4.8$ does not depend on density. The solid lines indicate fits according to the numeric model (see text), closely reproducing the characteristic behavior of the contrast. 
0.6 for $\omega_{\text {ex }}$ are included. For the case of highest density $\left(1.38 \times 10^{12} \mathrm{~cm}^{-3}\right)$ the agreement is within $50 \%$, which indicates failure of the infinite-range approximation. Note that the determination of $\Delta_{0}$ necessitates a good knowledge of the DLS and the density shift (see below). The simple theoretical model provides an excellent understanding of the measurements and allows for an optimization of the relevant experimental parameters towards a drastic increase of the coherence time.

Improvement of the coherence time requires simultaneous optimization of conditions (i) and (ii). While $\omega_{\mathrm{ex}} / \Delta_{0}$ can easily be increased with density, $\omega_{\mathrm{ex}} /\left(\pi \gamma_{c}\right)$ can only be increased by lowering the temperature. The mean trap frequency is therefore reduced to $2 \pi \times 51 \mathrm{~Hz}$ while cooling the ensemble to a temperature of $100(20) \mathrm{nK}$ at a mean density of $0.3 \times 10^{12} \mathrm{~cm}^{-3}$. In such an optimized configuration, $\omega_{\mathrm{ex}} /\left(\pi \gamma_{c}\right)$ is increased to 9.6 while preserving $\Delta_{0} / \omega_{\text {ex }}$ at 20.8. For these conditions, Fig. 2 shows the population transfer $P$ for a variable interrogation time of 0-35 s within an extended constant hold time. Although the fringes are quickly washed out due to the instability of the absolute frequency, the contrast persists over many seconds. The $1 / e$ coherence time is $21 \mathrm{~s}$ and complete loss of coherence occurs at $26 \mathrm{~s}$. A reduction of the contrast due to asymmetric atom loss can be neglected since we measure a 38 s differential lifetime of $|\uparrow\rangle$ and $|\downarrow\rangle$. The measured contrast is well reproduced by the numerical model without parameter fitting, including the nonexponential shape of the contrast reduction.

The presented analysis of spin self-rephasing requires precise knowledge of both the mean-field density shift and the DLS. To determine these values, we perform measurements of the absolute frequency for various atomic densities in three different trap configurations with mean trap frequencies of $2 \pi \times\{51,70$, and 112$\} \mathrm{Hz}$. The

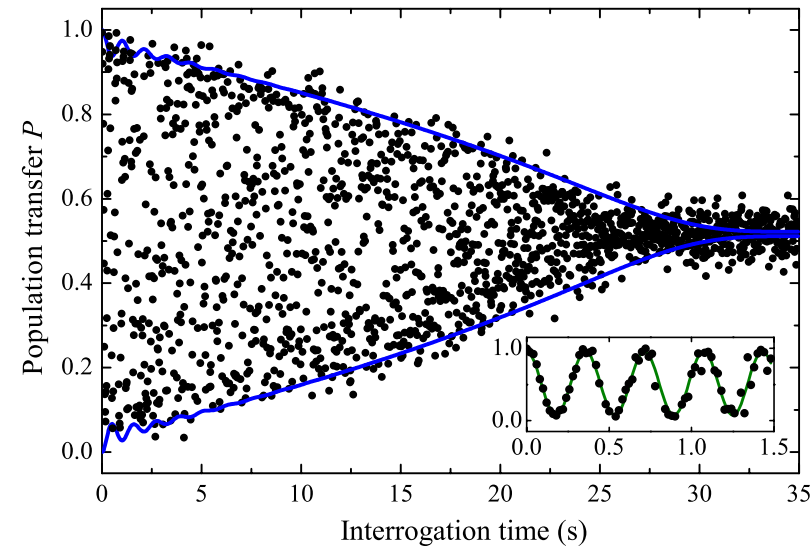

FIG. 2 (color online). Population transfer $P$ in a Ramsey sequence optimized for long coherence time (see text). The inset shows clear fringes for the first $1.5 \mathrm{~s}$. However, they wash out due to frequency noise after some seconds. Nonetheless the contrast remains well visible and a $1 / e$ coherence time of $21 \mathrm{~s}$ is obtained, a dramatic increase over previous experiments. The solid line represents a solution of the numeric model. measured frequency shifts with respect to the undisturbed transition are presented in Fig. 3(a). All three configurations show a clear linear dependence on the mean density. The fitted slopes lie within $10 \%$ of $\gamma / 2 \pi=-0.52 \mathrm{~Hz} /$ $\left(10^{12} \mathrm{~cm}^{-3}\right)$ and agree well with the predicted value of $-0.48 \mathrm{~Hz} /\left(10^{12} \mathrm{~cm}^{-3}\right)$ [29]. The DLS can be obtained from an extrapolation of this frequency shift to zero density. Figure 3(b) shows this value as a function of the ensemble averaged light intensity. We find a linear dependence on the intensity with $\delta \alpha / 2 \pi=-2.2(3) \mathrm{Hz} /$ $\left(\mathrm{kW} \mathrm{cm}{ }^{-2}\right)$, which constitutes the first direct measurement of the ${ }^{87} \mathrm{Rb}$ clock shift at $1064 \mathrm{~nm}$. This value confirms a first principle calculation [30] yielding $-2.4 \mathrm{~Hz} /$ $\left(\mathrm{kW} \mathrm{cm}^{-2}\right)$. The extrapolation to zero intensity results in a residual shift of 5.2(6) Hz. This value is in excellent agreement with the quadratic Zeeman shift of 5.2(1) Hz at the magnetic offset field of 95(1) $\mathrm{mG}$ which is determined from the $\Delta m_{F}=1$ transition. The presented extrapolation procedure will allow for an absolute measurement of the ${ }^{87} \mathrm{Rb}$ clock frequency in an optical trap.

Finally, we present the frequency stability of our measurements and analyze its principal noise contributions. Thereby, we can infer the potential benefit of long coherence times for a microwave frequency standard using an optically trapped ensemble in a purpose-built setup. To determine the noise contributions, the frequency is measured by Ramsey spectroscopy in a similar configuration to Fig. 1(d). The interrogation time was set to $350 \mathrm{~ms}$ coinciding with the first contrast revival. The detuning of $-8.4 \mathrm{~Hz}$ was chosen such that the mean population transfer is $P \approx 0.5$ on the central Ramsey fringe. The population transfer $P$ was recorded in 215 consecutive measurements at a cycle time of $28.1 \mathrm{~s}$. The number of measurements was limited to $\approx 2 \mathrm{~h}$ at night during standstill of the nearby tram line. Figure 4 shows the overlapping Allan standard deviation [31] for the frequencies extracted from the population transfer. The data follow a near-white
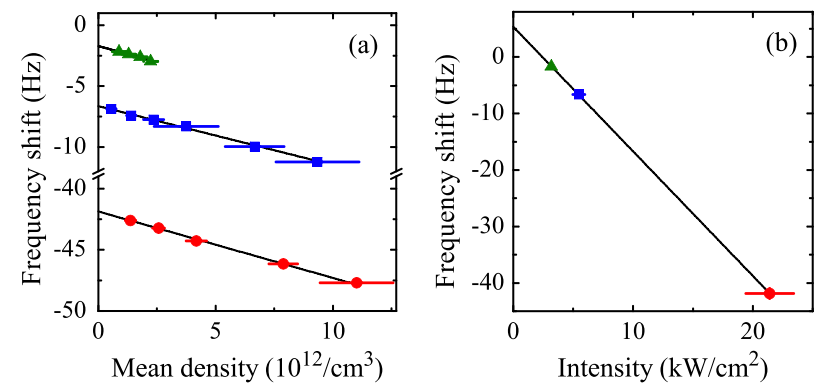

FIG. 3 (color online). Measurement of the relevant frequency shifts compared to the zero field transition. Three trap configurations with mean trap frequencies of $2 \pi \times 51 \mathrm{~Hz}$ (green triangles), $2 \pi \times 70 \mathrm{~Hz}$ (blue squares), and $2 \pi \times 112 \mathrm{~Hz}$ (red circles) were investigated. (a) Frequency shift as a function of the mean density (statistical error bars). The extrapolation to zero density yields a residual shift, plotted in (b) as a function of the ensemble averaged light intensity for each trap (estimated uncertainties). The slope of a linear fit determines the DLS. 


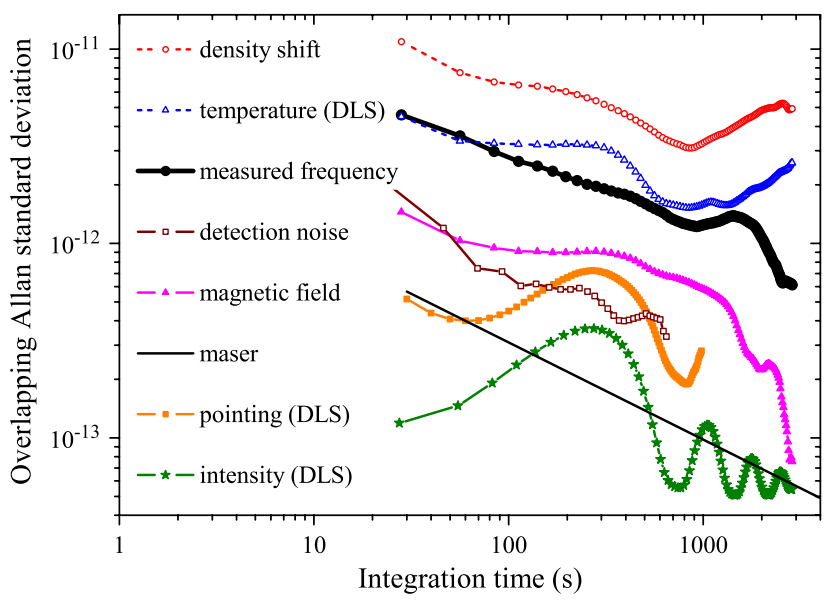

FIG. 4 (color online). Stability analysis based on the overlapping Allan standard deviation. The frequency measurement (solid black dots) yields a stability of $4.6 \times 10^{-12}$ at one shot, corresponding to $2.4 \times 10^{-11}$ at $1 \mathrm{~s}$. All relevant noise sources are measured independently (see text) and are shown here as frequency noise. The periodic modulation in some of the curves reflects the air-conditioning cycle of $10 \mathrm{~min}$.

frequency noise behavior of $2.4 \times 10^{-11} \tau^{-1 / 2}$, equivalent to $4.6 \times 10^{-12}$ at one shot.

For our parameters, the quantum projection noise limit is $8 \times 10^{-13} \tau^{-1 / 2}$, showing that our measurements are limited by technical noise. Seven relevant noise sources are measured independently and their frequency equivalents are plotted in Fig. 4: The maser is not limiting our measurements as its typical noise contribution including the Dick effect shows. The detection noise is $1 \%$ at one shot. Both of these effects decrease with a higher duty cycle and may be suppressed substantially by the prolonged interrogation time due to SSR. The DLS fluctuates if intensity or pointing of the trapping beams changes, which were measured as $6 \times 10^{-5}$ and $0.3 \mu \mathrm{m}$ at one shot, respectively. The magnetic field contribution, determined to be $0.1 \mathrm{mG}$ at one shot with an external Hall probe, is significant due to the lack of shielding. The contributions of mean-field density noise and DLS noise due to temperature fluctuations are overestimated, since their independent measurement is influenced by additional noise of the absorption imaging.

In summary, the effect of SSR was investigated in an optically trapped ensemble of ${ }^{87} \mathrm{Rb}$. By engineering the spin self-rephasing, we have demonstrated a coherence time of $21 \mathrm{~s}$ - the longest ensemble coherence time reported so far. The shifts and noise contributions to a frequency measurement were studied systematically and a short-term stability of $2.4 \times 10^{-11} \tau^{-1 / 2}$ was reached. We estimate the achievable stability in an optimized setup including magnetic field shielding, shallower trapping, and a tenfold increase in temperature determination. Assuming an increased duty cycle with an interrogation time of $21 \mathrm{~s}$ within a cycle time of $24 \mathrm{~s}$, we predict an achievable stability of $8 \times 10^{-14} \tau^{-1 / 2}$. This rivals the stability range of most atomic fountain clocks [1] in a potentially much more compact setup. The proven generality of the giant coherence time due to SSR makes it a promising candidate for application in optical lattice clocks and ensemble-based quantum information.

We acknowledge support from the Centre for Quantum Engineering and Space-Time Research (QUEST), the Institut Francilien de Recherche sur les Atomes Froids (IFRAF), and the Danish National Research Foundation Center for Quantum Optics (QUANTOP).

*kleinebuening@iqo.uni-hannover.de

Current address: Instituto de Ciencias Nucleares, UNAM, Circuito Exterior, Ciudad Universitaria, 04510 México, D.F., México.

[1] R. Wynands and S. Weyers, Metrologia 42, S64 (2005).

[2] C. W. Chou et al., Phys. Rev. Lett. 104, 070802 (2010).

[3] A. D. Ludlow et al., Science 319, 1805 (2008).

[4] Note recent progress in optically trapped Bose-Einstein condensates as reported in P. A. Altin et al., arXiv:1011.4713.

[5] K. Hammerer, A. S. Sørensen, and E. S. Polzik, Rev. Mod. Phys. 82, 1041 (2010).

[6] D. N. Matsukevich et al., Phys. Rev. Lett. 97, 013601 (2006).

[7] Y. O. Dudin et al., Phys. Rev. Lett. 103, 020505 (2009).

[8] H. de Riedmatten et al., Phys. Rev. Lett. 97, 113603 (2006).

[9] C.-W. Chou et al., Science 316, 1316 (2007).

[10] S. B. Papp et al., Science 324, 764 (2009).

[11] B. Zhao et al., Nature Phys. 5, 95 (2008).

[12] R. Zhao et al., Nature Phys. 5, 100 (2008).

[13] A. Derevianko and H. Katori, Rev. Mod. Phys. 83, 331 (2011).

[14] H. Katori et al., Phys. Rev. Lett. 91, 173005 (2003).

[15] K. Beloy et al., Phys. Rev. Lett. 102, 120801 (2009).

[16] P. Rosenbusch et al., Phys. Rev. A 79, 013404 (2009).

[17] A. Derevianko, Phys. Rev. Lett. 105, 033002 (2010).

[18] R. Chicireanu et al., Phys. Rev. Lett. 106, 063002 (2011).

[19] Y. Sagi, I. Almog, and N. Davidson, Phys. Rev. Lett. 105, 093001 (2010).

[20] Y. Sagi, I. Almog, and N. Davidson, Phys. Rev. Lett. 105, 053201 (2010).

[21] H. J. Lewandowski et al., Phys. Rev. Lett. 88, 070403 (2002).

[22] C. Deutsch et al., Phys. Rev. Lett. 105, 020401 (2010).

[23] K. Gibble, Phys. Rev. Lett. 103, 113202 (2009).

[24] K. Gibble, Physics 3, 55 (2010).

[25] J. N. Fuchs, D. M. Gangardt, and F. Laloë, Phys. Rev. Lett. 88, 230404 (2002).

[26] G. Kleine Büning et al., Appl. Phys. B 100, 117 (2010).

[27] F. Ramirez-Martinez et al., IEEE Trans. Ultrason. Ferroelectr. Freq. Control 57, 88 (2010).

[28] G. Reinaudi et al., Opt. Lett. 32, 3143 (2007).

[29] We use a $a_{\uparrow}=94.55 a_{B}, a_{\Downarrow}=100.76 a_{B}$ from a coupled channel analysis by E. Tiemann (private communication).

[30] A. Derevianko (private communication); see also [17].

[31] D. Howe, D. Allan, and J. Barnes, in Proceedings of the 35th Annual Frequency Control Symposium, Philadelphia, PA, 1981 (IEEE, New York, 1981), pp. 669-716. 